\title{
A Distribution Development for Wavelet - Transform - Based Image Denoising and, it's Presentation to Document Image Binarization
}

\author{
Rana Quadri ${ }^{1}$ | Qurratul Aini ${ }^{2}$ | Sumaya Tazeen ${ }^{3}$ \\ ${ }^{1}$ Dept ECE, Research Scholar, Shri JJTU university, Rajasthan, India \\ ${ }^{2}$ Dept ECE, Research Scholar, Shri JJTU university, Rajasthan, India \\ ${ }^{3}$ Dept ECE, Assistant Professor, SWCET, Hyderabad, Telangana, India
}

\section{To Cite this Article}

Rana Quadri, Qurratul Aini and Sumaya Tazeen, "A Distribution Development for Wavelet - Transform - Based Image Denoising and, it's Presentation to Document Image Binarization", International Journal for Modern Trends in Science and Technology, 6(8S): 20-22, 2020.

\section{Article Info}

Received on 16-July-2020, Revised on 15-August-2020, Accepted on 25-August-2020, Published on 28-August-2020.

\section{ABSTRACT}

To recognize picture highlights from "significant level clamors, we propose to misuse the spatial connections between wavelet coefficients by supplanting the thresholding procedure with a dispersion procedure". "The proposed smoothing technique is stronger to commotion when applied to profoundly tainted pictures".

\section{INTRODUCTION}

"Wavelet based picture denoising strategies have pulled in broad interests in the course of the most recent decade". "Donoho et. al. [3] first proposed to evacuate/stifle clamor by thresholding of wavelet coefficients". "The basic supposition that is basic what's more, natural a wavelet coefficient is treated as commotion and set to zero on the off chance that it is under a preset edge". "Something else, the coefficient is kept or somewhat adjusted". "To evaluate edge as exact as could reasonably be expected, various (adaptive plans have been proposed (see for example [1][2])". "While the Wavelet Transform-Threshold Inverse Transform (WTTI) strategies have been effective over broad tests, the supposition that one can recognize commotion from signal exclusively dependent on coefficient extents is abused when commotion levels are higher than signal sizes". "Under this high clamor situation, the spatial setup of neighbouring wavelet coefficients can play a significant job in clamor signal arrangements". "Signs will in general structure significant highlights (for example straight lines, bends), while loud coefficients disperse haphazardly". "In the current work, we misuse this spatial conflation of wavelet coefficients, joined with coefficient sizes, to order and stifle loud coefficients". "Specifically, we propose to supplant the second step of the Wavelet Change Thresholding-Inverse Transform (WTTI) strategies with a dispersion procedure, which iteratively alters wavelet coefficients with the end goal that the consistency among neighbouring coefficients is boosted (Section 2. I)". "In Segment 3 , we apply both a WTTI strategy and the proposed Wavelet Transform-Diffusion-Inverse Transform (WTDI) strategy to report picture binarization". "Tentatively, we show that the WTDI technique is more powerful to high commotion levels". 


\section{ADAPTIVE IMAGE DENOISING}

The proposed technique follows the overall three-advance wavelet based denoising strategies (see Figure I):

1. Figure the wavelet change ofthe clamor signal

2. Alter the wavelet coefficients;

3. Reverse change utilizing the adjusted coefficients.

"We use MATLAB wavelet bundle to complete wavelet (converse) changes in stages 1 and 3 . In sync 2". "We adjust the coefficients by augmenting the spatial consistency among neighbouring coefficients". "The layout of this dissemination procedure is given beneath". "In following subsections, we quickly audit the dissemination procedure and allude to [5][6] for point by point reports".

\subsection{The Diffusion Process}

"The first term, referred to as the local variance is the weighted sum of the Mahalanobis distances between $\mathrm{p}(\mathrm{x})$ and it neighbors". "Given fixed $\mathrm{g}(\mathrm{xn})$, minimizing this term will reduce the variations between neighboring coefficients". "The second term, which is the negated entropy of $g(x n)$, prevents $g(x n)$ to the over-segmented". " $X$ is the userspccified "scale factor" controlling the relative weights of these two terms".

$$
\left\{\begin{array}{l}
g^{(k)}\left(\mathbf{x}_{\mathbf{n}}\right)=\frac{1}{2} \exp \left\{-\lambda \mathcal{M}_{n}\left(\mathbf{p}^{(k)}(\mathbf{x}), \mathbf{p}\left(\mathbf{x}_{\mathbf{n}}\right), \mathbf{c}\left(\mathbf{x}_{\mathbf{n}}\right)\right)\right\} \\
z(\mathbf{x})^{(k+1)}=\frac{\sum_{\mathbf{x}_{\mathbf{n}} \in \mathbf{R}(\mathbf{x})} g^{(k)}\left(\mathbf{x}_{\mathbf{n}}\right)\left|v_{33}\left(\mathbf{x}_{\mathbf{n}}\right) z\left(\mathbf{x}_{\mathbf{n}}\right)^{(k)}-\left(\mathbf{x}-\mathbf{x}_{\mathbf{n}}\right) v_{\mathbf{x} 3}\left(\mathbf{x}_{\mathbf{n}}\right)\right|}{\sum_{\mathbf{x}_{\mathbf{n}} \in \mathbf{R}(\mathbf{x})} g^{(k)}\left(\mathbf{x}_{\mathbf{n}}\right) v_{33}\left(\mathbf{x}_{\mathbf{n}}\right)}
\end{array}\right.
$$

\section{EXPERIMENTAL RESULTS}

"In this segment, we apply the proposed technique to smooth uproarious pictures, and contrast it and three other picture smoothing techniquesfor example the Gaussian channel, the Median channel, and the Wavelet Threshold technique".

\subsection{Image Denoising}

"In this investigation, we utilize genuine pictures (Figure 4, with Gaussian commotion intentionally included) to test the exhibitions of diverse smoothing strategies". "It is appeared in Figure 5 (columns 1 and 2, see inscriptions), that the proposed WTDI technique can adequately smooth the clamor and still protect helpful highlights". "For instance in face pictures (sections 1,2), the clamor on faces are evacuated, while the facial highlights (eyes, mouth nose) are very much safeguarded" "The surfaces on lady's garments (segment 3), comers and edges (sections 4,s) are additionally kept". "Interestingly, the WTTl strategy (line 3) smothers clamor at the expense of obscured picture highlights". "The Gaussian channel (line 4) aimlessly stifles all high recurrence parts (both commotion and picture highlights)". "At last, the Median channel (line 5) produces blocky antiquities in smoothed pictures".

\subsection{Document Image Binarization}

In this trial, we utilize engineered archive pictures to analyze the exhibitions of various smoothing strategies:

1. Manufactured Noise: We include Gaussian ( $\mathrm{U}=$ $50,100,150$ separately) and rash commotion $(\mathrm{J}=$ $0.1)$ to two unique report pictures.

2. Picture Smoothing: The boisterous pictures are smoothed with four distinctive smoothing strategies.

3. Picture Binarization: The smoothing yields are binarized with a worldwide thresholding plan.

4. Execution estimation: By utilizing the Modified HausdotlT Distance (MHD) presented in [4], we measure the contrasts between the perfect binarization yields and the real binarization yields of various techniques. The MHD measures the normal spatial separation between two arrangements of focuses and approaches zero when two pictures become indistinguishable. "It is appeared in Figure 2 and Table 1, that the proposed strategy delivers the most honed and the most clear binarization results and compares to the most minimal binarizations mistakes under fluctuating clamor levels". "Figures 3 and Table 2 show comparative outcomes for Chinese characters".

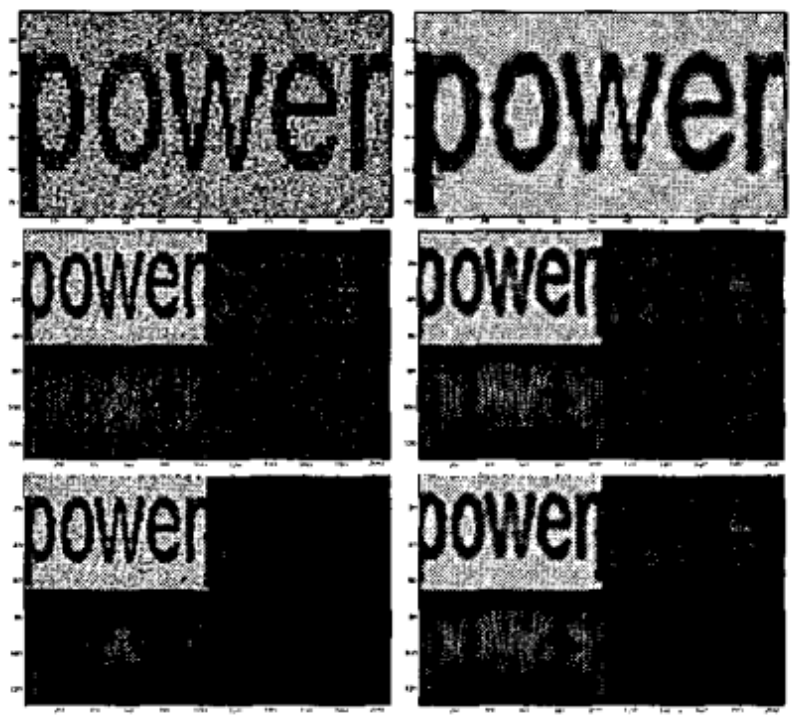

Figure 1: Wavelet

Transform-Diffusion-lnverreTransform. Upper lefl to bonom right: (a) Input noisy image; (b) Denoised image; (c) Wavelet Transform Coefficients (level I): four sub-images correspond to the apProximation 
(Upper lefl) and horizontal (upper fight), venical (bottom lee) and diagonal details @onom right); (d)

Wavelet 'Transform Coefficients (level 2); (e) Wavelet Diffused Coefficients (level 1); (0 Wavelet Diffused Coellicients (level 2)

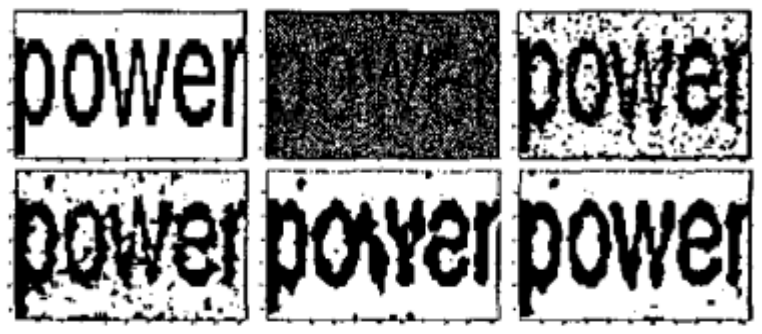

Figure 2: Binafizalion images with different smoothing methods

\begin{tabular}{|c|c|c|c|c|}
\hline$\sigma$ & WaveDiffuse & WaveThrd & Gaussian & Median \\
\hline 50 & $\mathbf{0 . 0 3 6 6}$ & 0.0874 & 0.3401 & 0.0463 \\
100 & $\mathbf{0 . 1 3 1 6}$ & 0.2239 & 0.6049 & 0.4314 \\
150 & $\mathbf{0 . 2 5 7 3}$ & 0.3267 & 0.8561 & 0.7703 \\
\hline
\end{tabular}

Table 1 : MHD of "power" for different smoothing methods under various noise levels

\section{CONCLUSIONS}

In the current work, we demonstrated that a dissemination procedure can recognize and smother clamor more viably than a thresholding process for both regular scene and archive picture denoising. The quality of our technique credits to the utilization of coplanar frameworks which portray spatial relationships of wavelet coefficients. In future work, we plan to test the appropriateness of our wavelet-based dissemination process in settings of edge identification and picture division.

\section{REFERENCES}

[1] S, Chang, 8, yu, and M. vetterli, lmge denoising via lossy $\mathrm{e}(\mathrm{lm}$. pression and wavelet thresholding, In In pmceedings of the IEEE International Conferee on pages 6w07, 1997,

[2] S. Chang, B. Yu, and M. Venerli. Spatially adaptive wavelet thresholding with context modeling for image demising. In In PmceedingJ of the IEEE Inrernalional Co+ence on Image Pmcessing, pages 535- 539, 1998.

[3] D. L. Danaho and 1. M. Johnstone. Ideal spatial adaptation via wavelet shrinkage. Biomelrikn, 81:425455. 1994.

[4] M. Dubusson and A. Jain. A modified haudorffdistance for object matching. In Pmceedings ofthe Inrernalionol CoQfewnce on Pottern Recognition. pages 566-568, Jerusalem. Israel, Oct 1994.

[5] L. Fan, L. Fan, and C. Tan. Adaptive Image Smoathing Using Coplanar Matrix and Its Application to Dwument Image Binarization. Inlernarionnl Journal on Doeirment Analysis and Recognition.

[6] L. Fan, L. Fan. and C. 'fan. Adaptive lnwe Resfomion Using Stmclure Tensors. 